\title{
IPAG - collaboration interprofessionnelle également en matière de cybersanté
}

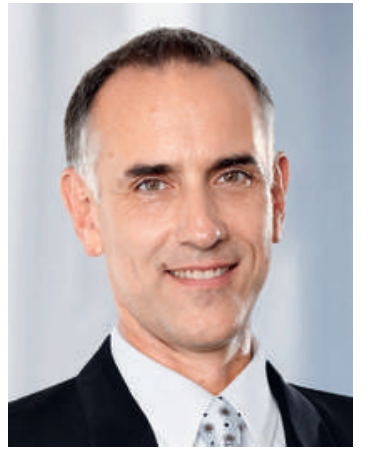

En avril 2014, huit associations professionnelles du domaine de la santé - pharmaSuisse, l'Association suisse des infirmiers et infirmières (ASI), ChiroSuisse, l'Association suisse des ergothérapeutes (ASE), l'Association suisse des diététicien-ne-s diplômé-e-s ES/ HES (ASDD), physioswiss et la FMH - ont fondé le Groupe de travail interprofessionnel pour le dossier électronique du patient (IPAG DEP).

Cette coopération, qui représente une avancée remarquable également du point de vue international, a suscité beaucoup d'intérêt lors de la Rencontre Consultative de toutes les organisations médicales germanophones de juin
Pour cela, les outils électroniques peuvent offrir une aide précieuse, à condition qu'ils soient centrés sur les patients et adaptés aux nécessités du quotidien. Avec ce constat, l'IPAG DEP veut profiter de ses connaissances des processus thérapeutiques et les intégrer dans le développement du dossier électronique du patient, afin d'avancer de la manière la plus ciblée possible.

La tâche principale de l'IPAG DEP consiste donc à identifier, coordonner et comparer les attentes et les exigences des différents groupes professionnels et à les réunir dans une solution soutenue par tous. Sa réflexion doit se concentrer sur les contenus dont découlera naturellement l'objectif visé, à savoir une architecture fidèle au principe de «form follows function».

Même si les débuts demandent pas mal de préparation et d'engagement de la part des différents fournisseurs de prestations, le groupe de travail interprofessionnel permettra de

\section{Plusieurs associations professionnelles du domaine de la santé collaborent pour que le dossier électronique du patient soit adapté aux besoins concrets.}

dernier. En s'associant, les huit associations professionnelles concernées veulent contribuer à l'évolution de la cybersanté en apportant leur expertise professionnelle. L'IPAG DEP aimerait en effet promouvoir activement le dossier électronique du patient qu'il considère comme un instrument essentiel pour soutenir des processus thérapeutiques interdisciplinaires qui soient centrés vers les patients.

Avec la spécialisation et la complexité accrue des trajectoires, la communication entre les différents maillons de la chaîne de traitement prend toujours plus d'importance, et le

\section{La cybersanté doit soutenir les différents groupes professionnels tout au long du traitement des patients.}

patient attend de toutes les personnes impliquées qu'elles coordonnent entre elles leurs interventions, de nature thérapeutique, infirmière, médicale ou autre, pour que le traitement dans son ensemble soit le plus efficace possible. La collaboration interprofessionnelle devra être orientée d'abord sur des soins de qualité élevée et sur le bénéfice des patients. concevoir le DEP de manière efficace et de cibler des solutions concrètes. Mais l'IPAG DEP évitera aussi les frictions les plus importantes et les retards tels qu'ils peuvent apparaître lors de consultations successives des acteurs concernés.

Lors de sa première séance, le groupe de pilotage de l'IPAG DEP a demandé de commencer les travaux sur les contenus, avec deux sujets complexes, les «rapports électroniques de sortie» et la «cybermédication», conformément aux priorités énoncées par eHealth Suisse. Ces sujets seront traités de manière ciblée, à chaque fois sous la conduite d'une des organisations plus importantes. Une des tâches définies de l'IPAG DEP consiste également à rester en contact permanent avec eHealth Suisse pour harmoniser et coordonner les avancées.

Nous sommes persuadés que la création de l'IPAG DEP est la concrétisation d'une excellente idée, dont le succès ne se fera pas longtemps attendre, et confirmera ainsi le bien-fondé de la démarche. Et pour tous les protagonistes, il est absolument clair que la cybersanté doit avant tout être au service des patients et de tous les soignants.

Dr Gert Printzen, membre du Comité central de la FMH, responsable du département eHealth - informatique et documentation médicales / Produits thérapeutiques 